\title{
Correction to: T1 mapping for liver function evaluation in gadoxetic acid-enhanced MR imaging: comparison of look-locker inversion recovery and B1 inhomogeneity-corrected variable flip angle method
}

\author{
Ji Eun Kim ${ }^{1} \cdot$ Hyun Ok Kim ${ }^{2} \cdot$ Kyungsoo Bae $^{1} \cdot$ Dae Seob Choi $^{1} \cdot$ Dominik Nickel $^{3}$ \\ Published online: 29 July 2019 \\ (C) European Society of Radiology 2019
}

Correction to: European Radiology (2019) 29:3584-3594

https://doi.org/10.1007/s00330-018-5947-4

The original version of this article, published on 22 March 2019, unfortunately contained a mistake. The following correction has therefore been made in the original: Affiliations 1 and 2 were presented incorrectly. The corrected version is given below.

Publisher's note Springer Nature remains neutral with regard to jurisdictional claims in published maps and institutional affiliations.

The online version of the original article can be found at https://doi.org/ $10.1007 / \mathrm{s} 00330-018-5947-4$

Hyun Ok Kim

galleonok@hanmail.net

1 Department of Radiology, Gyeongsang National University College of Medicine and Gyeongsang National University Hospital, 79

Gangnam-ro, Jinju 52727, Republic of Korea

2 Department of Internal Medicine, Gyeongsang National University College of Medicine and Gyeongsang National University Hospital, 79 Gangnam-ro, Jinju 52727, Republic of Korea

3 Siemens Healthineers, Erlangen, Germany 